\title{
PERTURBATION OF A STURM-LIOUVILLE OPERATOR BY A FINITE FUNCTION
}

\author{
RICHARD C. GILBERT
}

If $T_{1}$ is a self-adjoint operator and $V$ is a bounded self-adjoint operator in a Hilbert space and if $T_{2}=T_{1}+V$, then Theorem 1 of [2] states that

$$
\lim _{\tau \rightarrow \infty} \tau^{2}\left[S\left\{R_{2}(i \tau)-R_{1}(i \tau)\right\}+S\left\{R_{1}(i \tau) V R_{1}(i \tau)\right\} \mid=0\right.
$$

provided

$$
\left\||V|^{1 / 2} R_{1}(i \tau)\right\|_{2}=O\left(\tau^{-\alpha}\right) \quad \text { as } \quad \tau \rightarrow \infty, \quad \text { where } \alpha>1 / 2 .
$$

Here $R_{i}(z)$ is the resolvent of $T_{i},\|\|_{2}$ is the Schmidt norm, and $S$ stands for trace. From (1) various trace formulas for differential operators may be obtained. In [2] the condition (2) was verified for the situation in which $T_{1}$ is defined in $L^{2}[0, \infty)$ by the ordinary differential operator $L=-D^{2}$ and the boundary condition $u(0)=0$, and $V$ is the operator of multiplication by $p(x)$, where $p$ is real, continuous, bounded, and absolutely integrable on $[0, \infty)$. Recently M. G. Gasymov [1] derived trace formulas for the case that $L=-D^{2}+q$, where $q(x) \rightarrow \infty$ as $x \rightarrow \infty$, and $p(x)$ is a finite ${ }^{1}$ function. Gasymov's article suggests that condition (2) is valid for the case considered by him. It is the purpose of this article to show that if $p$ is a finite function and if $T$ is bounded below, then (2) holds in fact, whatever the behavior of $q$ at infinity is. The method employed is similar to that used by B. M. Levitan [3] for the study of the spectral function of $L$.

Theorem. Let $q$ be a real-valued continuous function on $[0, \infty)$. Let $T$ be a self-adjoint operator in $L^{2}[0, \infty)$ defined by $L=-D^{2}+q$ and the boundary condition $u^{\prime}(0)=0$. (If $L$ is in the limit circle case at infinity, a boundary condition at infinity is also included.) Let $p(x)$ be a realvalued continuous function on $[0, \infty)$ which vanishes for $x>A$. If $T$ is bounded below, $\left\||V|^{1 / 2} R(i \tau)\right\|_{2}=O\left(\tau^{-3 / 4}\right)$ as $\tau \rightarrow \infty$, where $V$ is the operator of multiplication by $p$, the norm is the Schmidt norm and $R(z)$ is the resolvent of $T$.

Presented to the Society, April 27, 1964; received by the editors May 7, 1964.

2 By a finite function is meant a function which vanishes outside some bounded set. 
Proof. If $\psi(x, \lambda)$ is the solution of $L u=\lambda u, u(0)=1, u^{\prime}(0)=0$, and if $\rho(\lambda),-\infty<\lambda<\infty$, is the spectral function of $T$, it is shown in [2] that

$$
\left\||V|^{1 / 2} R(i \tau)\right\|_{2}^{2}=\int_{0}^{\infty}|p(x)| \int_{-\infty}^{\infty} \frac{|\psi(x, \lambda)|^{2}}{\lambda^{2}+\tau^{2}} d \rho(\lambda) d x .
$$

Assuming that $T$ is bounded below by 0 and using the finiteness of $p$, (3) can be written

$$
\left\||V|^{1 / 2} R(i \tau)\right\|_{2}^{2}=\int_{0}^{\Lambda}|p(x)| \int_{0}^{\infty} \frac{|\psi(x, \lambda)|^{2}}{\lambda^{2}+\tau^{2}} d \rho(\lambda) d x .
$$

If $q$ is extended evenly to the negative real numbers, then $\psi(x, \lambda)$ is extended evenly to the negative real numbers also, and it may be verified that $\psi(x, \lambda) \cos \sqrt{ } \lambda t$ is the solution of an initial value problem for a hyperbolic equation over the whole $(x, t)$-plane. $\psi(x, \lambda) \cos \sqrt{ } \lambda t$ may therefore be written in the form

$$
\begin{aligned}
\psi(x, \lambda) \cos \sqrt{ } \lambda t= & (1 / 2)[\psi(x+t, \lambda)+\psi(x-t, \lambda)] \\
& +(1 / 2) \int_{x-t}^{x+t} \psi(s, \lambda) w(x, t, s) d s
\end{aligned}
$$

where $w(x, t, s)$ is constructed from the Riemann function of the hyperbolic equation and is continuous in $(x, t, s)$ for $-\infty<x, t, s<\infty$.

Let $g$ be a real-valued function defined on $[0,1]$ having the following properties: $g \in C^{2}, g(0)=1, g(1)=g^{\prime}(0)=g^{\prime}(1)=0$. If we multiply both sides of (5) by $e^{-a t} g(t)$ and integrate from 0 to 1 , we obtain, after some changes of variables, a reversal of order of integration, and use of the equation $\psi(x, \lambda)=\psi(-x, \lambda)$,

(6) $\psi(x, \lambda) \int_{0}^{1} \cos \sqrt{\lambda t} e^{-a t} g(t) d t=\int_{0}^{x+1} F(x, s, a) \psi(s, \lambda) d s, \quad x \geqq 0$,

where

$$
\begin{aligned}
F(x, s, a)=(1 / 2) & {\left[e^{-a|s-x|} g(|s-x|)+e^{-a|s+x|} g(|s+x|)\right.} \\
& +\int_{|x-s|}^{1} w(x, t, s) e^{-a t} g(t) d t \\
& \left.+\int_{|x+s|}^{1} w(x, t,-s) e^{-a t} g(t) d t\right], \\
& 0 \leqq x \leqq 1,0 \leqq s \leqq 1-x ;
\end{aligned}
$$




$$
\begin{array}{rl}
F(x, s, a) & =(1 / 2)\left[e^{-a|\sigma-x|} g(|s-x|)+\int_{|x-s|}^{1} w(x, t, s) e^{-a t} g(t) d t\right] \\
& =0, \quad 1 \leqq x, 0 \leqq s \leqq x-1 ; \\
& =(1 / 2)\left[e^{-a|\sigma-x|} g(|s-x|)+\int_{|x-s|}^{1} w(x, t, s) e^{-a t} g(t) d t\right] \\
1 & 1 \leqq x, x-1 \leqq s \leqq x+1 .
\end{array}
$$

Since the map $f(\lambda)=\int_{0}^{\infty} \psi(x, \lambda) f(x) d x$ is an isometry of $L^{2}[0, \infty)$ onto $L^{2}(\rho)$, it follows from (6) that

$$
\begin{array}{rl}
\int_{0}^{\infty}\left|\psi(x, \lambda) \int_{0}^{1} \cos \sqrt{ } \lambda t e^{-a t} g(t) d t\right|^{2} & d \rho(\lambda) \\
& =\int_{0}^{x+1}|F(x, s, a)|^{2} d s .
\end{array}
$$

Two integrations by parts show that

$$
\begin{aligned}
\int_{0}^{1} g(t) \cos \sqrt{ } \lambda t e^{-a t} d t= & \frac{a}{a^{2}+\lambda}\left[1+\int_{0}^{1} g^{\prime}(t) e^{-a t} \cos \sqrt{ } \lambda t d t\right. \\
& -\frac{\sqrt{ } \lambda}{a^{2}+\lambda} \int_{0}^{1} g^{\prime \prime}(t) e^{-a t} \sin \sqrt{ } \lambda t d t \\
& \left.-\frac{\lambda}{a\left(a^{2}+\lambda\right)} \int_{0}^{1} g^{\prime \prime}(t) e^{-a t} \cos \sqrt{ } \lambda t d t\right]
\end{aligned}
$$

Hence, there is a number $a_{0}>0$ such that

$$
\int_{0}^{1} g(t) \cos \sqrt{ } \lambda t e^{-a t} d t \geqq \frac{a}{2\left(a^{2}+\lambda\right)} \text { for } \lambda \geqq 0 \text { and } a \geqq a_{0},
$$

and therefore

$$
\left|\int_{0}^{1} g(t) \cos \sqrt{ } \lambda t e^{-a t} d t\right|^{2} \geqq \frac{a^{2}}{4\left(a^{2}+\lambda\right)^{2}} \geqq \frac{a^{2}}{8\left(a^{4}+\lambda^{2}\right)} \text { for } \lambda \geqq 0, a \geqq a_{0} .
$$

It follows from (7) that

(8) $\frac{a^{2}}{8} \int_{0}^{\infty} \frac{|\psi(x, \lambda)|^{2}}{a^{4}+\lambda^{2}} d \rho(\lambda) \leqq \int_{0}^{x+1}|F(x, s, a)|^{2} d s, \quad a \geqq a_{0}$.

Multiplying (8) by $|p(x)|$, integrating from 0 to $A$, and assuming $A>1$, one obtains 


$$
\begin{aligned}
& \frac{a^{2}}{8} \int_{0}^{A}|p(x)| \int_{0}^{\infty} \frac{|\psi(x, \lambda)|^{2}}{a^{4}+\lambda^{2}} d \rho(\lambda) d x \\
(9) \leqq & \int_{0}^{1}|p(x)|\left\{\int_{0}^{1-x}|F(x, s, a)|^{2} d s+\int_{1-x}^{1+x}|F(x, s, a)|^{2} d s\right\} d x \\
& +\int_{1}^{A}|p(x)| \int_{x-1}^{x+1}|F(x, s, a)|^{2} d s d x, \quad a \geqq a_{0} .
\end{aligned}
$$

By use of the triangle inequality and the continuity of the functions involved, one may show that the right side of (9) is $O(1 / a)$ as $a \rightarrow \infty$. Letting $a=\tau^{1 / 2}$, it follows from (4) and (9) that $\left\||V|^{1 / 2} R(i \tau)\right\|_{2}$ $=O\left(\tau^{-3 / 4}\right)$. This proves the theorem in the case that $T$ is bounded below by zero.

Now suppose that $T \geqq-m, m>0$. Let $T_{1}=T+m E$, where $E$ is the identity. Then $T_{1}$ is bounded below by zero. Also, $T_{1}$ is an ordinary differential operator of the same type as $T$. It follows from what we have already shown that $\left\||V|^{1 / 2} R_{1}(i \tau)\right\|_{2}=O\left(\tau^{-3 / 4}\right)$. Since

$$
\begin{aligned}
R(i \tau) & \left.=R_{1}(i \tau) \mid E+m R(i \tau)\right\rfloor,\left\||V|{ }^{1 / 2} R(i \tau)\right\|_{2} \\
& \leqq\left\||V|^{1 / 2} R_{1}(i \tau)\right\|_{2}\|E+m R(i \tau)\| \\
& \leqq\left\||V|^{1 / 2} R_{1}(i \tau)\right\|_{2}(1+m / \tau) .
\end{aligned}
$$

Thus the theorem is completely proved.

REMARK. The situation in which $T$ is defined by more general boundary conditions or $L$ has singularities at both ends of the interval can be handled similarly.

\section{REFERENCES}

1. M. G. Gasymov, On the sum of the differences of the eigenvalues of two self-adjoint operators, Dokl. Akad. Nauk SSSR 150 (1963), 1202-1205. (Russian)

2. R. C. Gilbert and V. A. Kramer, Trace formulas for a perturbed operator, Duke Math. J. 30 (1963), 275-296.

3. B. M. Levitan, On the asymptotic behavior of the spectral function of a self-adjoint differential equation of second order and on expansion in eigenfunctions, Izv. Akad. Nauk SSSR Ser. Mat. 17 (1953), 331-364. (Russian)

California State College at Fullerton 